\title{
Three-year comparison of subcutaneous insulin pump treatment with multi-daily injections on HbA1c, its variability and hospital burden of children with type 1 diabetes
}

\author{
Wojciech Fendler • Anna Iza Baranowska • \\ Beata Mianowska $\cdot$ Agnieszka Szadkowska \\ Wojciech Mlynarski
}

Received: 20 July 2011/Accepted: 14 September 2011/Published online: 1 October 2011

(C) The Author(s) 2011. This article is published with open access at Springerlink.com

\begin{abstract}
Treatment with continuous subcutaneous insulin infusion (CSII) allows a large degree of treatment individualization and intensification in children with diabetes. The study's aim was to evaluate the impact of treatment with CSII on glycated haemoglobin level (HbA1c) in children with diabetes and investigate whether introduction of CSII is associated with an increased risk of acute complications of diabetes. Patients treated throughout the recruitment period exclusively with multiple daily injections (MDI) were matched for duration of diabetes and HbA1c level at baseline with patients treated exclusively with CSII in a 1:1 group ratio $(n=223$ and 231 for MDI and CSII, respectively). The CSII group showed lower HbA1c after the observation period $(7.98 \pm 1.38$ vs. $7.56 \pm 0.97 ; P=0.002)$. HbA1c variability measured as standard deviations of average values was also lower in the CSII group $(0.73 \pm 0.45$ vs. $0.84 \pm 0.54 ; P=0.049)$. The rate of hospitalization due to acute events was similar in both groups (14.7/100 vs. 14.0/100 person/years in the MDI and CSII group, $P=0.72$ ). Duration of hospital stay per year was on average 1.25 days shorter in the CSII group $(P=0.0004)$, but the risk of acute complications resulting in hospitalization did not differ between the groups (hazard ratio (HR) 1.16; 95\% confidence interval (95\% CI) $0.68-1.63$ ). The most significant risk factor for
\end{abstract}

Electronic supplementary material The online version of this article (doi:10.1007/s00592-011-0332-7) contains supplementary material, which is available to authorized users.

W. Fendler · A. I. Baranowska · B. Mianowska ·

A. Szadkowska · W. Mlynarski ( $\square)$

Department of Paediatrics, Oncology, Haematology

and Diabetology, Medical University of Lodz,

36/50 Sporna St., 91-738 Lodz, Poland

e-mail: wojciech.mlynarski@joslin.harvard.edu hospitalization due to acute complications was baseline HbA1c concentration (HR 1.25; 95\% CI 1.14-1.37). In conclusion, CSII treatment may improve glycemic control and reduce its variability. Change of MDI to CSII does not alter the risk of hospitalization and may reduce the annual duration of hospitalization in children with diabetes.

Keywords Insulin delivery systems .

Complications of diabetes - Paediatric diabetes .

Healthcare organization

\section{Introduction}

In Poland, the incidence of type 1 diabetes in the population below 15 years of age tripled during the last three decades $[1,2]$. During that time, new methods of diabetes management such as rapid and long-acting insulin analogues, continuous subcutaneous insulin infusion (CSII) and continuous glucose monitoring (CGM) systems have been implemented. The primary goals of these technologies were to improve metabolic control, decrease the frequency of acute and chronic diabetes complications and minimize the overall healthcare burden. Numerous studies have unequivocally shown that better metabolic control decreases chronic diabetes-related complications [3]. Although the quality of paediatric diabetes care has markedly improved, hospital visits related to acute complications still constitute a serious burden for the healthcare system [4]. Several studies in children and adolescents have shown improvements in terms of better metabolic control, reduced rates of complications and better quality of life [5-10]. Patients treated with CSII are not, however, free from acute complications, e.g. rates of diabetic ketoacidosis or hypoglycaemic events, and the hospital 
burden of diabetes in such patients is still considerable [11]. Moreover, some reports are in favour of additional precautions during the switching period, with regard to the type of insulin used, its dose and patient's age [12].

The aim of this study was to evaluate the impact of treatment with CSII on the metabolic control (expressed as glycated haemoglobin level-HbA1c) and its variability in children with diabetes. The study also investigated whether the introduction of CSII was associated with altered risk, or rates, of acute complications of diabetes in prolonged treatment.

\section{Patients and methods}

\section{Patients}

Throughout the observation period, the study centre treated $>90 \%$ of children with diabetes within the Lodz administrative region, inhabited by 2.6 million people. This was a prospective observational study based on a standardized computerized, manually curated database of discharge summaries of all patients with type 1 diabetes hospitalized at the study centre from January 2002 to December 2010. The number of patients treated within the centre increased from 520 to 632 throughout the study period. The centre's diabetes epidemiology database used in nationwide and regional trials $[2,13]$ covers all patients registered at the hospital discharge summaries database, which ruled out the possibility of missing patients with diabetes treated in the region. The CSII group was collected without any specific restrictions concerning the patients; the decision to introduce CSII was based on the patient's or their parents' willingness to change the treatment in view of more freedom in food intake and lifestyle, and on the clinical judgment of the physician. Clinical indications for CSII were high HbA1c levels (above 8-8.5\%), dawn phenomenon, frequent hypoglycaemia (at least two episodes per year of severe hypoglycaemia with need for intervention of another person or loss of consciousness), concomitant chronic illnesses or willingness to improve the patient's quality of life. The CSII group was collected between January 2002 and December 2008. Subsequent follow-up was continued until December 2010.

\section{Methods}

Patients treated throughout the recruitment period exclusively with multiple dose injections (MDI) were matched retrospectively for duration of diabetes and baseline $\mathrm{HbA} 1 \mathrm{c}$ level to the CSII group to ensure a 1:1 group ratio. Propensity score matching was attempted; it is a technique aimed at the elimination of group allocation bias through calculation of individual probability of being assigned to the treatment or control group on the basis of covariates associated with group allocation [14, 15]. However, as it would lead to imbalanced group numbers in terms of individual patients (Electronic Supplementary Material, Table 1), covariate matching was used instead. Criteria for inclusion into the analysis were set as following: type 1 diabetes diagnosis according to WHO criteria, lack of obesity ( $<90$ th centile of body mass index for age and sex), treatment with insulin with a mean daily dose $>0.3$ units/ $\mathrm{kg}$, age at onset greater than 12 months, duration of diabetes $>1$ year and presence of at least one anti-islet autoantibody at onset (islet cell antibody, anti-GAD, insulin autoantibody or IA2). Patients with type 2 diabetes, cystic fibrosis or confirmed monogenic diabetes were excluded from both groups. Patients who changed treatment from CSII to MDI, started CSII therapy prior to January 2002 or switched their treatment regimen twice during the observation period were not considered eligible for the analysis. Patients who were diagnosed with type 1 diabetes during the observation period but were not subsequently treated as inpatients or outpatients at the study Centre were excluded from the analysis $(n=118)$. During the follow-up, scheduled hospital admissions of patients with type 1 diabetes have been carried out every 2.5 year (to perform screening tests for chronic diabetic complications, common autoimmune diseases and for re-education). Only acute hospitalizations were considered in this analysis. Considering the access to the treatment, the cost of insulin treatment other than with long-acting insulin analogues was fully reimbursed by the state throughout the study period. Costs of insulin pumps and tubing for children were covered either by a non-profit organization (the Great Orchestra of Christmas Charity) or by the National Health Fund.

\section{Analysed outcomes}

The primary outcome of the study was the risk of acute hospitalization due to hypo- and/or hyperglycaemia and acute hospitalization for any reason. Hospitalization due to hypoglycaemia was defined as hospital admission due to: (1) symptoms typical for hypoglycaemia that improved after oral/parenteral glucose or parenteral glucagon administration or (2) documented low capillary/venous blood glucose concentration, both acknowledged/recognized by the admitting physician as requiring hospitalization because of the severity (severe hypoglycaemia) or recurrence of symptoms (i.e. due to gastrointestinal infection or insulin overdose). Hospitalization due to hyperglycaemia was defined as hospital admission due to: (1) ketoacidosis, (2) high blood glucose levels (over $250 \mathrm{mg} / \mathrm{dl}$ ) persisting for at least $12 \mathrm{~h}$ and not improving in the ambulatory setting or 
(3) incorrect glycaemic control as reflected by high HbA1c level assessed by the guiding diabetologist as not acceptable for the individual patient (typically over $8 \%$ depending on patient's age, history of experienced severe hypoglycaemia, concomitant illnesses and previous metabolic control). The MDI group used various combinations of long-acting analogues, NPH insulin, short-acting analogues or human insulin. No subgroup analyses based on insulin type used were possible, as changes were frequent, occurred multiple times in numerous patients and may have been dependent on the availability of particular insulin preparations.

Measured outcomes included the following: HbA1c level measured at the moment of CSII introduction or matching (for MDI-treated patients) and at the end of the observation period or censoring due to reaching the age of 18 years (mean HbA1c $8.05 \pm 1.30$ ); meeting the therapeutic goal of $<7.0 \%$ of HbA1c [16]; number and duration of hospital visits calculated for the whole observation period; time to first hospitalization due to acute causesfor this purpose, patients who reached 18 years of age or had no acute visits since enrolment before the study closing date were treated as censored observations.

\section{Laboratory measurements}

\section{Glycated haemoglobin measurements}

Throughout the whole study period, the laboratory method for the assessments of $\mathrm{HbA}_{1 \mathrm{c}}$ was consistent. $\mathrm{HbA}_{1 \mathrm{c}}$ assays were performed by ion-exchange high-performance liquid chromatography (HPLC) using the Bio-Rad VARIANT ${ }^{T M}$ Hemoglobin Alc Program (Bio-Rad Laboratories, Inc. Hercules, California, USA). The VARIANT ${ }^{T M}$ Hemoglobin Alc Program has been certified by the National Glycohemoglobin Standardization Programme (NGSP) as meeting the DCCT standard. The within-run coefficient of variation (CV) determined by the manufacturer was $1.05 \%$ for people without diabetes and $0.94 \%$ for people with diabetes; the between-run CV was 1.61 and $1.16 \%$ for people without diabetes and for people with diabetes, respectively. Blood samples were collected with HbAlc Capillary Collection System (Bio-Rad Laboratories, Inc. Hercules, California, USA) and analysed according to the manufacturer's protocol.

\section{C-peptide measurements}

To determine whether residual beta cell function was similar between the two groups, C-peptide levels were measured at baseline whenever possible using radioimmunoadsorption assays (IRMA-C-PEP, CIS Bio International). Both intraand interassay $\mathrm{CV}$ for this assay equalled 4.9 and $5.1 \%$, respectively, according to the manufacturer. Minimum detectable C-peptide level equalled $0.036 \mathrm{mmol} / \mathrm{ml}$, and its concentrations in a group of 40 healthy individuals were within the $0.35-1.17 \mathrm{mmol} / \mathrm{ml}$ range.

\section{Statistical analysis}

Analysis of covariance (ANCOVA) models were used for the evaluation of mean $\mathrm{HbA} 1 \mathrm{c}$ and its variability (expressed as standard deviations-SD) with adjustment for duration of diabetes. Correlations were evaluated using Pearson's correlation coefficient. Final HbA1c values were additionally converted to percentile values of patients treated within the centre to assess the profile of each group with respect to metabolic profile of the clinic. For comparisons of percentiles of HbA1c and the number of hospital days per patient-year, the Mann-Whitney U test was applied with medians and interquartile ranges (IQRs) used for result presentation. The log-rank test was used for uncorrected comparison of survival without acute complications depending on treatment type. To adjust for covariates, a multivariate proportional hazard regression analysis including data on patient's sex, initial HbA1c, age and duration of diabetes at enrolment was developed. A $P$ value of $<0.05$ was considered to be the threshold of statistical significance.

\section{Results}

Comparison of baseline characteristics

The study group consisted of 454 patients-231 on CSII and 223 on MDI. Baseline study group characteristics are given in Table 1. The CSII group used pumps manufactured by Medtronic (Paradigm series and older devices; 67\%), Roche (Accu-Chek; 24\%) and Deltec (Cozmo; 9\%). Insulin pumps were loaded with insulin analogues $(81 \%)$ or human insulin (19\%). No patient used sensor-augmented pumps as they only became available in Poland in 2009. Significant differences in between-group comparisons were observed only in terms of age at initial visit. No differences in gender distribution, metabolic control or duration of diabetes were observed between the study groups as a result of covariate matching. Glycated haemoglobin levels showed moderate, significant correlations with patients' age $(r=0.21 ; P<0.0001)$ and duration of diabetes $(r=0.36 ; P<0.0001)$.

Comparisons of metabolic outcomes

Post-study HbA1c values deteriorated only in the MDI group, but the increase in $\mathrm{HbA} 1 \mathrm{c}$ was not statistically 
Table 1 Baseline characteristics of the study group

\begin{tabular}{lllr}
\hline & MDI & CSII & \multicolumn{1}{c}{$P$} \\
\hline Males/females & $126 / 97$ & $129 / 102$ & 0.8877 \\
Age at enrolment [years] & $14.13(10.82-16.18)$ & $10.20(7.17-13.15)$ & $<0.0001$ \\
Duration of diabetes at enrolment [years] & $2.46(0.70-5.53)$ & $2.43(1.47-5.21)$ & 0.3607 \\
Daily insulin dose [U/kg] & $0.81(0.63-1.09)$ & $0.95(0.53-1.57)$ & 0.2770 \\
$\begin{array}{l}\text { C-peptide level [ng/ml] } \\
\quad \text { normal range }>0.35 \mathrm{ng} / \mathrm{ml})\end{array}$ & $0.19(0.10-0.33)$ & $0.17(0.12-0.26)$ & 0.4101 \\
$\quad$ HbA1c at enrolment [\%] & $7.4(6.6-8.3)$ & $7.2(6.7-8.1)$ & 0.6001 \\
$\quad($ normal range 4.3-5.7\%) & & & \\
\hline
\end{tabular}

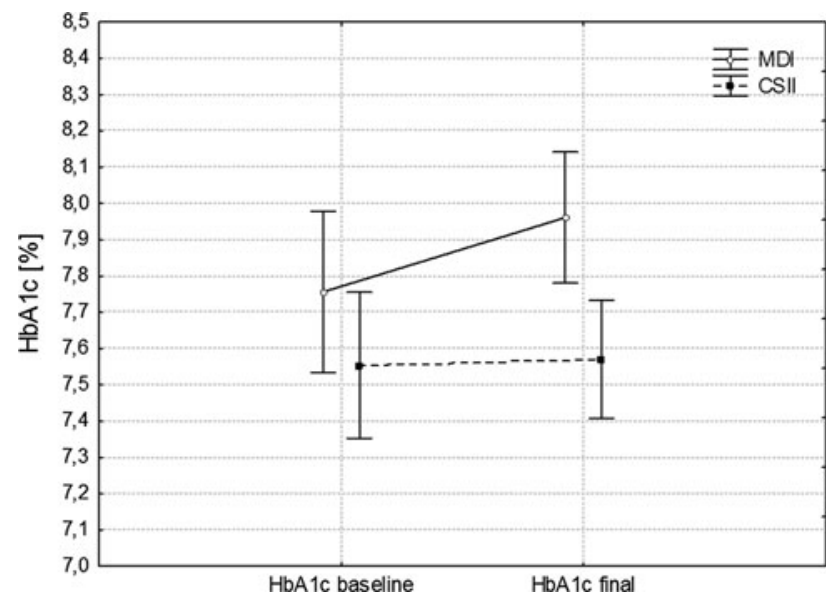

Fig. 1 Differences in age-adjusted glycated haemoglobin (HbA1c) levels at baseline and at the end of the observation period in patients treated with multiple daily injections (MDI) or continuous subcutaneous insulin infusion (CSII). The difference between MDI and CSIItreated patients was significant at the end of the observation period $(P=0.01)$. Data are depicted as means with $95 \%$ confidence intervals

significant [0.21\% (95\% CI -0.20 to 0.61$)$; Fig. 1]. Mean post-study HbA1c levels were statistically higher in MDI than in CSII-treated patients $(7.98 \pm 1.38$ vs. $7.56 \pm 0.97$, respectively; $P=0.002$ ); this difference persisted after adjustment for patient's age at enrolment and duration of diabetes $(P=0.01$; Fig. 1$)$.

Variability of metabolic control differed significantly between the groups-SD of HbA1c equalled $0.73 \pm 0.45$ in the CSII group in comparison with $0.84 \pm 0.54$ in MDItreated individuals ( $P=0.049$; Fig. 2$)$.

Percentile comparisons performed to ascertain the relative improvement of $\mathrm{HbAlc}$ showed that the CSII group ranked significantly better, with median percentile of final HbAlc equal to 45\% (IQR 24-68\%) in comparison with $59 \%$ (IQR 29-81\%) noted in MDI-treated individuals $(P=0.001)$.

The percentage of patients reaching the therapeutic goal of $<7 \%$ of $\mathrm{HbA} 1 \mathrm{c}$ equalled $32.9 \%$ in the CSII and $25.7 \%$ in the MDI group $(P=0.12)$.

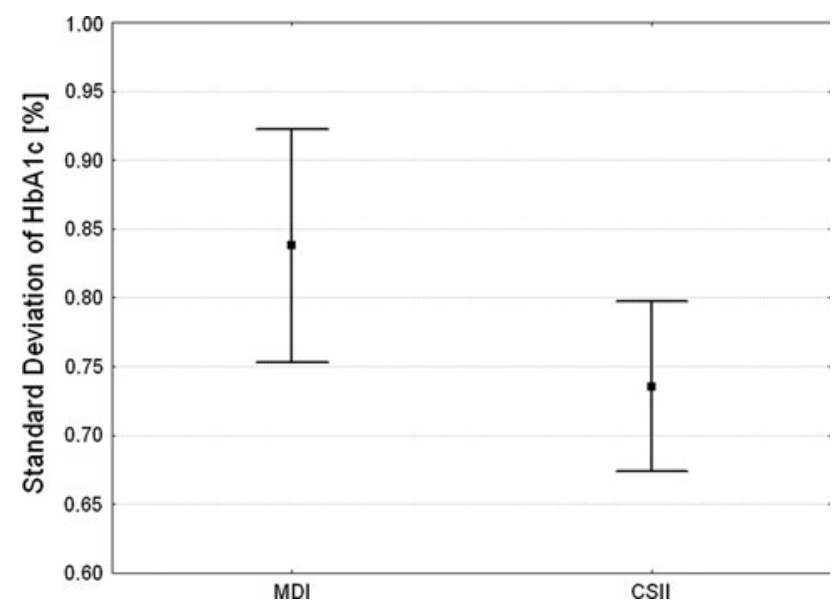

Fig. 2 Differences in standard deviation of glycated haemoglobin levels in the studied groups. The difference between the multiple daily injections (MDI) and subcutaneous insulin infusion (CSII) groups was of borderline statistical significance $(P=0.049)$. Data are depicted as means with $95 \%$ confidence intervals

Comparisons of hospital burden in children treated with CSII or MDI

Mean observation time was 3.05 years \pm 1.74 . The MDI group was observed for a total of 674 and the CSII group for 714 patient-years. The number of acute visits in the department equalled 99 and 100 for MDI- and CSII-treated patients, respectively. Rates of emergency hospital visits were similar in patients treated with MDI (14.7/100 patientyears) versus CSII (14.0/100 patient-years; $P=0.72)$. Total duration of hospital stays due to emergency admissions did not differ significantly between the groups $(P=0.94)$, although patients treated with CSII had a significantly lower number of total hospital days per year than the MDI group [3.5 (2.1-5.67) vs. 4.76 (2.46-9.63) $P=0.0004)]$. Throughout the observation period, 60 patients required at least one hospitalization due to acute causes. In $69.2 \%$ of cases, the underlying problem was hyperglycaemia and in $17.3 \%$, severe hypoglycaemia. Additional causes were identified in $13.5 \%$ of cases-most were related to infectious diseases $(n=14)$, urolithiasis 


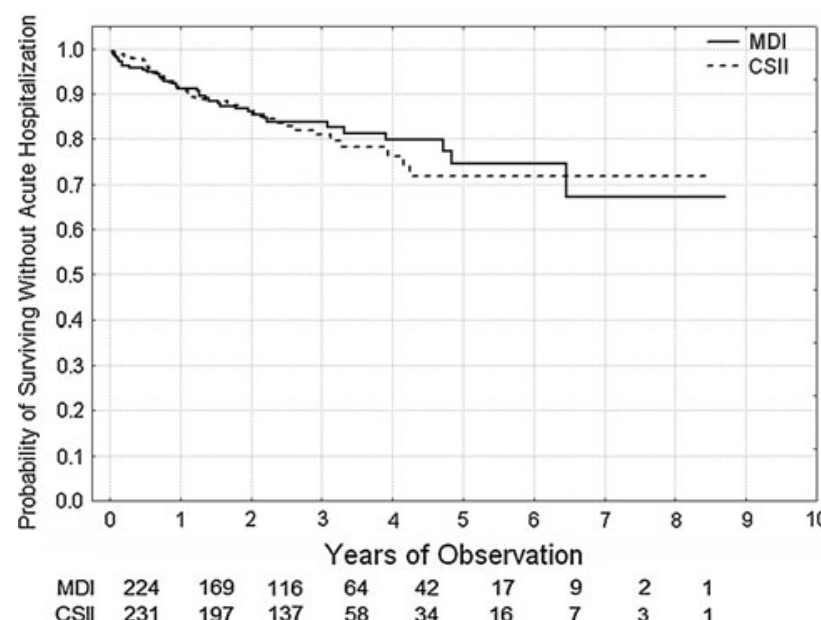

Fig. 3 Cumulative probability of survival without acute hospitalization of patients treated with multiple daily injections (MDI) or continuous subcutaneous insulin infusion (CSII). The risk of hospitalization did not differ between the studied groups $(P=0.77)$

Table 2 Multivariate Cox regression analysis of factors affecting the risk of acute hospitalization

\begin{tabular}{llll}
\hline Variable & $\begin{array}{l}\text { Hazard } \\
\text { ratio }\end{array}$ & $\begin{array}{l}\text { 95\% Confidence } \\
\text { Interval }\end{array}$ & $P$ \\
\hline Baseline HbA1c level [\%] & 1.25 & $1.14-1.37$ & 0.0001 \\
Male gender & 1.29 & $0.84-1.75$ & 0.2626 \\
Treatment with CSII & 1.16 & $0.68-1.63$ & 0.5438 \\
Age at enrolment & 1.01 & $0.94-1.08$ & 0.8270 \\
Duration of diabetes & 1.00 & $0.92-1.07$ & 0.9496 \\
Baseline C-peptide level & 1.31 & $0.30-5.78$ & 0.7236 \\
\hline
\end{tabular}

CSII continuous subcutaneous insulin infusion

$(n=4)$ or neurologic abnormalities [epilepsy, vertigo, headache or alcohol abuse $(n=4)$ ]. Univariate analysis did not show any difference in the risk of acute hospitalization based on treatment (log-rank $P=0.77$; Fig. 3). In multivariate Cox regression analysis, the risk of acute hospitalization depended only on initial HbAlc level. Treatment regimen, sex, age and duration of diabetes at enrolment were not statistically significant in this model (Table 2). Results did not differ when only acute hospitalization due to hypoglycaemic or hyperglycaemic episodes was considered as joint or separate endpoints. Adjusted hazard ratios for CSII treatment equalled 1.26 (95\% CI 0.04-2.48) for hospitalization due to hypoglycaemia, 1.30 (95\% CI 0.69-1.92) for hospitalization due to hyperglycaemia and 1.28 (95\% CI $0.73-1.83$ ) for hospitalization due to either of these causes. Duration of hospital stay due to acute causes did not differ significantly between the groups, although patients treated with CSII had a lower number of total hospital days per year (Table 3 ). The frequency of hospital visits did not differ between the groups;
Table 3 Duration of hospital stay and number of visits for any reason or emergency ones

\begin{tabular}{lcll}
\hline & MDI & CSII & $P$ \\
\hline $\begin{array}{l}\text { Days in hospital per } \\
\text { year }\end{array}$ & $4.76(2.46-9.63)$ & $3.50(2.11-5.67)$ & 0.0004 \\
$\begin{array}{l}\text { Days in hospital per } \\
\text { year due to } \\
\text { emergency visits }\end{array}$ & $0(0-0.47)$ & $0(0-0.43)$ & 0.9328 \\
\hline
\end{tabular}

median number of hospital visits per patient equalled 2 $(25-75 \% 1-3)$ in the MDI group and $2(25-75 \% 2-3)$ in the CSII group $(P=0.11)$.

\section{Discussion}

Benefits gained through CSII application included a slower deterioration of $\mathrm{HbA} 1 \mathrm{c}$ values, evidenced by an increasing difference in initial and final $\mathrm{HbA} 1 \mathrm{c}$ levels depending on CSII or MDI treatment. Curiously, baseline characteristics of the presented study group suggest that the analysed patients could have been in fact better controlled than those in the STAR 3 trial [9], (mean HbA1c levels $7.6 \pm 1.5$ vs. $8.5 \pm 0.6$ ) making it more difficult to obtain a statistically significant improvement of HbA1c. Stable HbA1c levels despite ageing and duration of diabetes observed in CSIItreated individuals in this trial do, however, mirror the beneficial effect of the STAR 3 participants. Final mean $\mathrm{HbA1c}$ levels in the paediatric group in STAR 3 equalled $7.7 \%$, whereas in the presented cohort the final mean HbA1c reached 7.6\%. Despite the methodological differences and application of different insulin pumps (the STAR 3 pumps were sensor-augmented), results confirming the overall benefit of CSII seem surprisingly convergent. Moreover, the difference in final $\mathrm{HbAlc}$ levels was similar to that reported in a recent meta-analysis by Monami et al. [17] who showed a $-0.3 \%$ reduction in favour of the CSII group. This may be interpreted as showing that CSII offers an advantage in terms of better metabolic control, but the improvement will seldom exceed $0.5 \%$ in long-term observation. Further improvements could theoretically be gained through the use of more sophisticated therapeutic approaches such as dual wave bolus (DWB) [18, 19]. However, due to the magnitude duration of observation, changing patient preferences in using DWB, the authors refrained from introducing this factor to stratify the CSII group.

Although CSII was shown to offer better metabolic control, this effect did not translate into the risk of acute hospitalizations. A lack of difference in this endpoint suggested that educational measures undertaken during the switching phase were adequate and did not result in 
shortened time to first hospital visit due to acute complications. Finally, rates of hospitalizations due to acute visits did not differ between the groups which confirmed that no additional risk of hypoglycaemia or ketoacidosis was created by CSII treatment, in contrast to earlier studies on that matter [20].

The obviously non-random allocation of patients is the main limitation of the study and a potential for bias, but the lack of differences in baseline HbAlc and duration of diabetes justified this approach and made the groups comparable. The authors performed covariate matching for baseline $\mathrm{HbA1c}$ and duration of diabetes which lead to balanced group numbers, apart from the age difference, which was adjusted for in multivariate comparisons. The perfect design - a randomized study - for a group of such magnitude would be unfeasible from an organizational point of view. Recently published results of an ambitious project on the use of sensor-augmented CSII (the STAR 3 trial) [9] covered 156 children allocated to either CSII or MDI. The observational approach with matching of MDI and CSII patients used in this study, although potentially biased by non-random selection, allowed the authors to include a study group of 454 individuals, yielding greater statistical power and robustness in terms of adjusted effects estimation. The fact that the differences in $\mathrm{HbAlc}$ persisted after correction for confounding factors further increased the credibility of presented findings.

Presented patients were followed for 3 years, which allowed the authors to evaluate the effect of CSII on hospitalization rates and variability of $\mathrm{HbA1c}$. The latter effect of CSII may be clinically important, as lower SD of HbA1c is a result of reduced rates of prolonged periods glycaemia maintained alternatively within the high or low concentration range. CSII treatment was already reported to reduce blood glucose variability in a meta-analysis authored by Pickup et al. [21]. Although variability of HbA1c is not yet considered an endpoint in diabetes trials, it was proven to increase the risk of long-term complications of diabetes in DCCT data analysis [22]. Therefore, interventions aimed at its reduction could be perceived as being potentially beneficial and therefore worthy of further interest.

Results of this study in terms of HbA1c changes due to CSII treatment were in line with those obtained by Jakisch et al. [23] who in a group of 434 matched pairs of patients with type 1 diabetes, showed an improvement of $\mathrm{HbA1c}$ during the first year of the study. In their study however, the difference disappeared after 3 years of observation, showing that the benefit could be attributed to the "novelty" effect. In the presented study, the effect seemed to persist throughout the observation period of similar duration (mean 3.05 years), possibly due to different educational approaches or changes in patients' ability to cope with diabetes and its management.
Persistent residual function of the beta cells could also contribute to better metabolic control in CSII-treated patients. However, as only $12 \%$ of analysed patients had C-peptide levels within normal range and no difference was detected between the groups, it seems very unlikely that residual beta cell function contributed significantly to the observed outcomes.

Over $30 \%$ of patients required at least one hospitalization due to acute complications during the observation period. This could result in a bias of study results, as the older patients may have left the group after shorter observation periods before developing acute complications, while younger ones could be observed for longer periods of time increasing their chances of acute hospitalization. However, the rate of acute hospitalizations per 100 patient/ years was nearly identical between CSII and MDI-treated patients, as was the case for risk of the first acute hospitalization, which allowed the authors to assume that the risk of being hospitalized due to acute conditions is the same with MDI or CSII treatment. CSII did, however, promote shorter overall hospital stays per year by nearly $25 \%$, which could result in an economic benefit from this type of treatment.

The rate of severe hypoglycaemia in an outpatient setting among children with diabetes is hard to estimate, as reported rates range widely from 6.63 events per 100 patient-years, reported by the PedPump study which included patients from Poland [24], to 62 per 100 patientyears reported earlier by Levine et al. [25]. In the Hvidore study group, which formulated the criteria for assessment of hypoglycaemic events used in the above-mentioned studies, the rate of severe hypoglycaemia equalled 22 per 100 patient-years [26]. Introduction of CSII treatment was shown to reduce the overall rate of hypoglycaemia [21] and was expected to result in a reduction in hospitalizations due to this complication in the studied group. The observed lack of differences in this outcome could result from one of the limitations of the presented study - the authors were unable to objectively collect and analyse data from outpatient clinics and match them with those from the hospital database. All patients and their parents could contact their attending diabetologists by phone and did so in critical situations including severe hypoglycaemic episodes, but precise recall of the overall and individual numbers of such episodes was not possible. As a result, the authors were unable to determine actual rates of hypoglycaemia not resulting in hospital admission. Hospitalizations due to hypoglycaemia, however, constituted $25 \%$ of all acute diabetes-related hospital visits in our study group, which is a similar value range as that noted in an earlier study by Palta et al. [27] The authors reported a rate of 1.9 per 100 patient-years hospital admissions due to hypoglycaemic events in patients with diabetes aged 0-29, which 
amounted to $21 \%$ of all hospital admissions of their cohort. Thus, it seemed that the group presented within this study did not deviate significantly from those reported in previous reports in terms of hypoglycaemia incidence, with no evident benefits gained through the introduction of CSII.

Confirmation of the fact that baseline HbAlc was the main risk factor for hospitalization due to acute complications may be one with considerable clinical implications. This association was previously reported in epidemiologic studies on children with diabetes $[25,27,28]$. One could therefore assume that patients with initially worse metabolic control should be a group of particular interest for the physician, as any intervention leading to the improvement of $\mathrm{HbA1c}$ could yield beneficial effects in terms of reducing the risk of acute hospitalizations. The percentage of patients reaching the therapeutic goal of ADA for $\mathrm{HbA1c}(<7.0 \%)$ was considerably greater in the presented study in both CSII and MDI groups than in the STAR 3 cohort. The difference was particularly evident in MDI individuals ( $25.7 \%$ vs. $10 \%$ reported by STAR 3), undermining the beneficial effect of CSII and suggesting suboptimal diabetes management in the MDI group in the cited study.

\section{Conclusions}

Treatment with CSII contributed towards better metabolic control of diabetes in terms of $\mathrm{HbA} 1 \mathrm{c}$ level and reduction in its variability. Application of CSII did not affect the rate of acute hospitalizations or the risk of acute hospitalization in comparison with MDI in children with diabetes but was associated with a shorter duration of annual in-hospital stay.

Acknowledgments The study was performed as part of the TEAM project financed by the Innovative Economy Programme of the European Union coordinated by the Foundation for Polish Science.

Open Access This article is distributed under the terms of the Creative Commons Attribution Noncommercial License which permits any noncommercial use, distribution, and reproduction in any medium, provided the original author(s) and source are credited.

\section{References}

1. Jarosz-Chobot P, Deja G, Polanska J (2010) Epidemiology of type 1 diabetes among Silesian children aged 0-14 years, 1989-2005. Acta Diabetol 47:29-33

2. Jarosz-Chobot P, Polanska J, Szadkowska A, Kretowski A, Bandurska-Stankiewicz E, Ciechanowska M et al (2011) Rapid increase in the incidence of type 1 diabetes in Polish children from 1989 to 2004, and predictions for 2010 to 2025. Diabetologia 54:508-515

3. The Diabetes Control and Complications Trial Research Group (1993) The effect of intensive treatment of diabetes on the development and progression of long-term complications in insulin-dependent diabetes mellitus. N Engl J Med 329:977-986

4. Estrada CL, Danielson KK, Drum ML, Lipton RB (2009) Hospitalization subsequent to diagnosis in young patients with diabetes in Chicago, Illinois. Pediatrics 124:926-934

5. Cohen O, Korner A, Chlup R, Zoupas CS, Ragozin AK, Wudi K et al (2009) Improved glycemic control through continuous glucose sensor-augmented insulin pump therapy: prospective results from a community and academic practice patient registry. J Diabetes Sci Technol 3:804-811

6. Muller-Godeffroy E, Treichel S, Wagner VM (2009) Investigation of quality of life and family burden issues during insulin pump therapy in children with Type 1 diabetes mellitus-a largescale multicentre pilot study. Diabet Med 26:493-501

7. Hoogma RP, Hammond PJ, Gomis R, Kerr D, Bruttomesso D, Bouter KP et al (2006) Comparison of the effects of continuous subcutaneous insulin infusion (CSII) and NPH-based multiple daily insulin injections (MDI) on glycaemic control and quality of life: results of the 5-nations trial. Diabet Med 23:141-147

8. Hanas R, Ludvigsson J (2006) Hypoglycemia and ketoacidosis with insulin pump therapy in children and adolescents. Pediatr Diabetes 7(suppl 4):32-38

9. Bergenstal RM, Tamborlane WV, Ahmann A, Buse JB, Dailey G, Davis SN et al (2010) Effectiveness of sensor-augmented insulinpump therapy in type 1 diabetes. N Engl J Med 363:311-320

10. Misso ML, Egberts KJ, Page M, O'Connor D, Shaw J (2010) Continuous subcutaneous insulin infusion (CSII) versus multiple insulin injections for type 1 diabetes mellitus. Cochrane Database Syst Rev CD005103

11. Danne T, Battelino T, Kordonouri O, Hanas R, Klinkert C, Ludvigsson $\mathrm{J}$ et al (2005) A cross-sectional international survey of continuous subcutaneous insulin infusion in 377 children and adolescents with type 1 diabetes mellitus from 10 countries. Pediatr Diabetes 6:193-198

12. Colino E, Alvarez MA, Carcavilla A, Alonso M, Ros P, Barrio R (2010) Insulin dose adjustment when changing from multiple daily injections to continuous subcutaneous insulin infusion in the pediatric age group. Acta Diabetol 47:1-6

13. Mianowska B, Fendler W, Szadkowska A, Baranowska A, Grzelak-Agaciak E, Sadon J et al (2011) $\mathrm{HbA}(1 \mathrm{c})$ levels in schoolchildren with type 1 diabetes are seasonally variable and dependent on weather conditions. Diabetologia 54:749-756

14. Rosenbaum PRRDB (1983) The central role of the propensity score in observational studies for causal effects. Biometrika 70:41-55

15. Robins JM, Mark SD, Newey WK (1992) Estimating exposure effects by modelling the expectation of exposure conditional on confounders. Biometrics 48:479-495

16. (2011) Executive summary: standards of medical care in diabetes-2011. Diabetes Care 34(suppl 1):S4-S10

17. Monami M, Lamanna C, Marchionni N, Mannucci E (2010) Continuous subcutaneous insulin infusion versus multiple daily insulin injections in type 1 diabetes: a meta-analysis. Acta Diabetol 47:77-81

18. Klupa T, Skupien J, Cyganek K, Katra B, Sieradzki J, Malecki MT (2011) The dual-wave bolus feature in type 1 diabetes adult users of insulin pumps. Acta Diabetol 48:11-14

19. O'Connell MA, Gilbertson HR, Donath SM, Cameron FJ (2008) Optimizing postprandial glycemia in pediatric patients with type 1 diabetes using insulin pump therapy: impact of glycemic index and prandial bolus type. Diabetes Care 31:1491-1495

20. Weissberg-Benchell J, Antisdel-Lomaglio J, Seshadri R (2003) Insulin pump therapy: a meta-analysis. Diabetes Care 26:10791087

21. Pickup J, Mattock M, Kerry S (2002) Glycaemic control with continuous subcutaneous insulin infusion compared with 
intensive insulin injections in patients with type 1 diabetes: metaanalysis of randomised controlled trials. BMJ 324:705

22. Kilpatrick ES, Rigby AS, Atkin SL (2008) A1C variability and the risk of microvascular complications in type 1 diabetes: data from the Diabetes Control and Complications Trial. Diabetes Care 31:2198-2202

23. Jakisch BI, Wagner VM, Heidtmann B, Lepler R, Holterhus PM, Kapellen TM et al (2008) Comparison of continuous subcutaneous insulin infusion (CSII) and multiple daily injections (MDI) in paediatric type 1 diabetes: a multicentre matched-pair cohort analysis over 3 years. Diabet Med 25:80-85

24. Danne T, Battelino T, Jarosz-Chobot P, Kordonouri O, Pankowska E, Ludvigsson J et al (2008) Establishing glycaemic control with continuous subcutaneous insulin infusion in children and adolescents with type 1 diabetes: experience of the PedPump Study in 17 countries. Diabetologia 51:1594-1601
25. Levine BS, Anderson BJ, Butler DA, Antisdel JE, Brackett J, Laffel LM (2001) Predictors of glycemic control and short-term adverse outcomes in youth with type 1 diabetes. J Pediatr 139:197-203

26. Mortensen HB, Hougaard P (1997) Comparison of metabolic control in a cross-sectional study of 2,873 children and adolescents with IDDM from 18 countries. The Hvidore Study Group on Childhood Diabetes. Diabetes Care 20:714-720

27. Palta M, LeCaire T, Daniels K, Shen G, Allen C, D'Alessio D (1997) Risk factors for hospitalization in a cohort with type 1 diabetes. Wisconsin Diabetes Registry. Am J Epidemiol 146:627636

28. Fritsch M, Rosenbauer J, Schober E, Neu A, Placzek K, Holl RW (2011) Predictors of diabetic ketoacidosis in children and adolescents with type 1 diabetes. Experience from a large multicentre database. Pediatr Diabetes 12(4 Pt 1):307-312 